Itinéraires Itinéraires

Littérature, textes, cultures

\title{
Expérimenter en langues, 2. L'intraduction : défiguration et valeur politique du langage
}

Experimenting in Tongues, 2. "Untranslating": Disfiguration and the Political Value of Language

\section{Mathias Kusnierz}

\section{OpenEdition}

\section{Journals}

Édition électronique

URL : http://journals.openedition.org/itineraires/7978

DOI : $10.4000 /$ itineraires.7978

ISSN : 2427-920X

\section{Éditeur}

Pléiade

Référence électronique

Mathias Kusnierz, «Expérimenter en langues, 2. L'intraduction : défiguration et valeur politique du langage », Itinéraires [En ligne], 2020-1 | 2020, mis en ligne le 23 septembre 2020, consulté le 14 novembre 2020. URL : http://journals.openedition.org/itineraires/7978 ; DOI : https://doi.org/10.4000/ itineraires.7978

Ce document a été généré automatiquement le 14 novembre 2020.

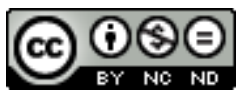

Itinéraires est mis à disposition selon les termes de la licence Creative Commons Attribution - Pas d'Utilisation Commerciale - Pas de Modification 4.0 International. 


\title{
Expérimenter en langues, 2. L'intraduction : défiguration et valeur politique du langage
}

\author{
Experimenting in Tongues, 2. "Untranslating": Disfiguration and the Political \\ Value of Language
}

Mathias Kusnierz

1 En 1983 paraît le numéro 15 de la revue TXT, intitulé Intraduction aux étrangers ${ }^{1}$. Plusieurs poètes réputés difficiles y sont publiés et traduits : Andrei Biely, Carlo Emilio Gadda, Charles Olson, Pier Paolo Pasolini, Edward E. Cummings et Gertrude Stein. Dans la réunion de ces écritures si diverses et le mot-valise qui donne son titre au numéro, un programme théorique se fait entendre : la poésie, c'est à la fois l'intraduisible et l'effort accompli pour le surmonter. La lecture du numéro révèle ensuite la teneur de cet effort : au moyen de multiples stratégies formelles, qu'elles soient typographiques, plastiques ou sonores, la traduction opère une défiguration sur le texte d'origine afin de forcer les résistances de la langue-source et de l'écriture (Ladmiral 1979: 14-22 ; $2014: 3-27,56-62$ ). Il s'agit en quelque sorte d'introduire de force la traduction dans des textes qui s'opposent à cette opération. L'intraduction est donc le nom de la traduction défigurante.

2 Je n'emploie pas ce mot - et les auteurs de TXT non plus, et pour cause ! - au sens que lui donne Pascale Casanova (1999 : 189-191, 322-328 ; 2015 : 51 ; Casanova et Samoyault 2005 : 139-150), à savoir la traduction qui introduit (ou importe) le texte étranger dans la langue du traducteur. Dans le concept que je propose de développer ici, on entend bien, comme chez Casanova, l'idée d'introduction mais celle-ci opère comme par effraction : il s'agit de traduire en dépit des résistances du texte. Dans le nom "intraduction» résonne donc également l'adjectif «intraduisible». Mais peut-être faut-il également prêter l'oreille au préfixe privatif seul et y oublier le in latin qui fait toujours de la traduction une introduction, une avancée vers et dans la langue d'autrui. L'intraduction serait aussi une non-traduction, qui ne renoncerait pas pour autant à faire jouer les 
langues les unes avec les autres en dépit de leur incommensurabilité (Ortega y Gasset [1940] 1964 : 434 ; Chiurazzi 2013).

3 Le comité éditorial de TXT donne suite à ce programme théorique dès le numéro suivant, avec un dossier réunissant des poètes de langue allemande : Gunter Falk, Ernst Jandl, Friederike Mayröcker, Oskar Pastior et Reinhard Priessnitz. Cinq ans plus tard, pour son numéro 24 intitulé D.D.R. Lyrik 1989, la revue traduit et publie dix poètes d'avant-garde vivant en République démocratique allemande: Jan Faktor, Stefan Döring, Eberhard Haefner, Bernd Igel, Andreas Koziol, Leonhard Lorek, Bert PapenfussGorek, Rainer Shedlinski, Michael Thulin et Ulrich Zieger. La réflexion sur la traduction poétique se double ici d'un programme politique puisque la revue publie des poètes qui ont éprouvé, pour la plupart, des difficultés à se faire publier ou à lire leurs textes en public. Le numéro 24 fait ainsi exister des écritures marginales et hétérodoxes, qui transforment l'usage du langage dans un contexte historique de répression sociale, d'autoritarisme politique et de contrôle des libertés publiques.

4 Dans cette étude, j'aborderai les textes (et leurs traductions par d'autres) d'Oskar Pastior, Ernst Jandl, Pierre Guyotat, James Joyce et Edward E. Cummings, ainsi que des traductions produites par Friedrich Hölderlin, Pierre Klossowski et Jacques Demarcq. Je réunis donc en un même corpus des textes polyglottes et des traductions qui butent sur de l'intraduisible. Textes originaux ou traduits, tous s'affrontent aux vertiges de la babélisation.

$5 \quad$ Ils ne forment bien sûr pas un corpus unifié, pour des raisons historiques qu'il n'est pas besoin de développer. Mais ils forment une constellation qui gravite autour de la revue TXT, puisque tous y ont été publiés ou commentés. Tous sont liés par l'enjeu des avantgardes poétiques des décennies 1960, 1970 et 1980, souvent qualifiées de «secondes avant-gardes historiques » (Prigent 1989: 9; 2004: 85-88). Dans le contexte contestataire de ces années, où l'efflorescence théorique le dispute aux prises de position politique, elles se donnent pour tâche de théoriser et d'accomplir un programme où l'expérimentation formelle porte avec elle le germe de la transformation politique. C'est le credo de la revue Change, animée par Jean-Pierre Faye dans les années 1960 et 1970 : l'évolution des formes anticipe et détermine celle de l'histoire (Faye 1968: 5-13). Quant à la revue TXT, elle a élaboré un programme théorique autour de l'idée des " grandes irrégularités de langage » que formule Georges Bataille (1955 : xxviII) dans la préface à Justine ou Les Malheurs de la vertu du Marquis de Sade. Pour son comité de rédaction, ces irrégularités de langage sont un facteur de dissidence politique, étant entendu que lorsque nous nous soustrayons aux manières communes de parler par l'écriture ou la lecture, nous nous arrachons également aux idéologies que porte ce langage commun (Prigent 1994 : 15-21; 2009 : 235-250 ; 2014 : 35).

6 Pourquoi s'intéresser à ces textes aujourd'hui ? D'abord parce que certaines de ces traductions, en forçant les digues de l'intraduisibilité, ont fait date et ont permis la découverte de poètes majeurs. Parmi elles, quelques-unes peuvent désormais être qualifiées de "traductions historiques", pour reprendre une expression de Berman (1984: 251) sur les traductions de Sophocle et Pindare par Hölderlin: à savoir des traductions qui marquent leur époque par leur singularité ou parce que, d'une manière ou d'une autre, elles constituent une œuvre à part entière et revêtent la même importance que le texte original. Ensuite parce qu'elles jettent une lumière oblique sur l'œuvre originale de leurs auteurs et leur rapport au langage: les traductions de 
Cummings par Jacques Demarcq éclairent les rapports de détermination mutuelle entre manipulations linguistiques et jeux typographiques dans Les Zozios ou Suite Apollinaire. Enfin ces poétiques glossolaliques, bien que la plupart des textes étudiés ici aient paru entre les années 1960 et 1980, sont encore nos contemporaines. Non seulement elles nous apportent un socle conceptuel pour comprendre les formes expérimentales qui en sont les héritières, mais en nous rappelant à la dimension babélienne de la production littéraire elles touchent, comme par ricochet, les questions politiques qui agitent aujourd'hui la littérature comparée et la traductologie, à savoir la pensée postcoloniale (Bhabha 1994) et les rapports de domination qui structurent la littérature mondiale (Casanova 1999 et 2015).

\section{Défiguration et intraduisible}

7 La défiguration, qui sera l'un des concepts centraux de mon étude, est l'une des stratégies formelles développées par les auteurs de TXT dans ce contexte théorique. D'une part, elle désigne le geste linguistique qui cherche à dépasser l'intraduisible en refigurant la langue par l'usage de détours formels : calembours, jeux typographiques et sonores, dysorthographie, etc. D'autre part, elle est l'effort stylistique qui arrache la langue aux normes de la communication courante au profit d'une autre langue, c'est-àdire la langue réduite à sa matière première, son pur balbutiement ou son bégaiement. Pour le Deleuze de Critique et clinique, un écrivain «est un étranger dans sa propre langue : il ne mélange pas une autre langue à sa langue, il taille dans sa langue une langue étrangère et qui ne préexiste pas » (1993: 138). Je voudrais souligner ici les termes qu'utilise Deleuze : inventer une langue consiste à la découper au sein d'une langue préexistante et plus vaste, comme on découpe un plan dans un volume. Or ce découpage spatial devient, dans la langue, le découpage rythmique du phrasé qui articule ensemble les mots du texte. Deleuze écrit que « chaque mot se divise, mais en soi-même [...] et se combine, mais avec soi-même » (139). La langue, alors, forme une «litanie de disjonctions» (24), jusqu'à devenir «le dehors du langage», que Deleuze définit comme «de pures visions [...] qui se rapportent encore au langage en tant qu'elles constituent un but ultime, un dehors, un envers, un dessous, tache d'encre ou écriture illisible ", et dans lesquels « les mots font silence » (141-142). Or ce silence des mots n'est autre que leur méconnaissabilité, si l'on me permet ici d'user d'un néologisme, c'est-à-dire de leur défiguration. D'après Christian Prigent, elle ouvre par et dans la langue, « un trou - de non-savoir, d'impossible, d'innommable» (2015: 13) dans le contour stabilisé des choses, du monde et de l'expérience. Ce trou désigne précisément l'inquiétude que provoque l'œuvre littéraire lorsqu'elle vise à arracher son lecteur aux assignations que lui fait la langue courante et qu'elle déploie sa puissance "contrethéologique ${ }^{2} »$ (Barthes $\left.1984: 66\right)$.

De fait, la défiguration produit aussi de l'intraduisible. Elle malmène et désarticule la langue commune; elle la rend incompréhensible, illisible, impartageable, singulière, intraduisible. Je pense ici à Pierre Guyotat ou à Artaud écrivant que « tout langage vrai est incompréhensible»(Prigent 1992: 4). Dans son livre La Défiguration, Évelyne Grossman écrit que ce concept désigne le « mouvement de déstabilisation qui affecte la figure» (2004: 9). Elle est « une force de création qui bouleverse les formes stratifiées du sens et les réanime » (7). Elle consiste, selon Artaud, à « substituer aux formes figées 
de l'art des formes vivantes et menaçantes" (1987: 37). En interdisant l'herméneutique, la défiguration menace les formes de conservation du sens.

\section{Trou(v)er une langue}

9 Je reviens ici à la manière dont Christian Prigent s'est approprié le concept de défiguration dans TXT. Pour le poète, l'aventure de la littérature d'avant-garde invite "à vivre toute langue comme étrangère et donc à refonder un langage (à "trouver une langue", disait Rimbaud ${ }^{3}$ ) pour re-présenter cette représentation " qu'est la langue ordinaire (Rimbaud 2009: 346; Prigent 1992: 5). J'en tire deux conclusions. Premièrement, la littérature d'avant-garde a fondamentalement à voir avec la traduction dans la mesure où elles font toutes deux naviguer l'écrivain et son lecteur entre plusieurs langues. Deuxièmement, le travail poétique consiste à défigurer la langue commune : à en modifier les règles, les contours et les formes pour élaborer une langue refigurée, de façon à ce que les locuteurs la vivent comme nouvelle, familière (elle ressemble à celle d'origine) et étrangère, identifiée et méconnaissable à la fois. En somme, il faut que la traversée de cette langue refigurée fasse apparaître un écart sensible avec la langue courante, c'est-à-dire que le lecteur la devine sans la comprendre et que, dans cette lecture mal assurée et désormais en péril, il soit luimême mis en danger par cette langue dont il devient le locuteur mental. Comme si la langue défigurée-refigurée était une maladie que son contact inoculait au lecteur, à la manière du langage-virus imaginé par Burroughs dans The Naked Lunch et la trilogie (The Soft Machine, The Ticket That Exploded et Nova Express) puis théorisé dans The Electronic Revolution (1974: 15-38, 57-62 ; 1979: 26-31; Prigent 1989:113-114). Le langage que travaille Pierre Guyotat dans Progénitures et celui que met au point Klossowski dans sa traduction de l'Énéide relèvent chacun d'une défiguration.

Chez Guyotat, l'écriture est, d'emblée, une écriture traductrice : elle nous prive de nos coordonnées linguistiques usuelles pour nous faire entrer dans un autre espace langagier, fondé sur le génotexte plutôt que le phénotexte (Kristeva 1969: 278-289), c'est-à-dire sur la manifestation du corps physique dans le corps de la langue plutôt que sur la capacité abstraite à la symbolisation linguistique. Voici comment Guyotat ouvre Progénitures ainsi que l'espace linguistique dans lequel il propose de faire entrer le lecteur :

(la Grande-Caissière du bordel du maitre B, de nuit, à un ouvrier entrant :)

- «l' jiarret boueux, l' orbit' empossiàrée, ta poitrin' m' trembler, l' comptoir caiss',

ta mâchoir', a'c, mes chieveux chiauds,

d' ö, l' hors bordel, banlioue?, Franç'?, m' dehanchier, gars, tes cent-un kilogs chantants, quaqu' banquet celest'?

d' quà ta faç' mâchiurée d' chiarogn' rat - l' relent l' tueur en refaufiler sa lam' d'dans sa taill'! -,

l' mouchiassat t' en rentrer s' assoupir dedans tes narin'! » (Guyotat $2000: 11$ )

Ces quelques lignes d'ouverture font exister chacun des points cardinaux de ce nouvel espace linguistique : dysorthographie et syntaxe malmenée; accents qui travaillent à déformer le rythme normé de la langue par un souffle adverse fondé sur les apocopes et les élisions ; exposition de la mécanique du corps à travers la nomination éclatée de ses organes et notamment de ceux qui assurent la marche, la parole et le souffle, de sorte que le texte dessine une équivalence entre ces trois phénomènes, entre la respiration et 
la marche toutes deux conçues comme une rythmique. L'organologie, chez Guyotat, donne le tempo de la respiration de la langue.

Dans sa traduction de l'Énéide, Klossowski suit rigoureusement l'ordre des mots latins, de manière à respecter :

l'aspect disloqué de la syntaxe, propre non seulement à la prose mais à la prosodie latine dans la traduction d'un poème où c'est précisément la juxtaposition volontaire des mots (dont le heurt produit la richesse sonore et le prestige de

l'image) qui constitue la physionomie de chaque vers. (Klossowski [1964] 2015 : 15)

Il restitue également les raccourcis de la langue latine qu'une traduction française doit développer et expliciter pour être idiomatique, ainsi que les particularités de la syntaxe latine. Aussi la phrase française épouse-t-elle ici les structures de la langue latine de sorte que cette dernière travaille, de l'intérieur, la première. La traduction de Klossowski ressemble à celle que théorisait Antoine Berman, à savoir une traduction qui fait entendre la langue-source dans la langue-cible et dans laquelle l'étranger résonne comme un écho proche et lointain (1984:16-24, 286-289).

Creusé dans le flanc gigantesque de la roche Euboïque, l'antre

où larges conduisent cent accès vers cent ouvertures

d'où se ruent autant de voix, réponses de la Sibylle?

Ils étaient arrivés sur les seuils, lorsque la Vierge : « Voici l'heure

d'interroger la fatalité », dit-elle: «le dieu! Voici le dieu!» Tandis que telles

paroles elle profère,

devant les portes subitement ce n'est plus le même visage, ni la même couleur

ni peignés ne demeurent ses cheveux; mais la poitrine haletante,

d'une rage de fauve son sein se gonfle : plus grande de taille elle semble

et d'un accent qui n'a rien d'une mortelle, du souffle touchée de la puissance

déjà proche du dieu4. (Virgile, trad. Klossowski [1964] 2015b : 180-181)

On voit distinctement, ici, que Klossowski recherche l'homologie des structures syntaxiques, le raccourci, la littéralité la plus immédiate et, sur le plan lexical, la plus grande simplicité. Cela n'empêche pas Klossowski d'utiliser un grand nombre de néologismes archaïsants, comme « haste » (lance) ou « bipenne » (char à deux chevaux, nom féminin) ${ }^{5}$. Ce mode de traduction, en restituant les structures latines à l'intérieur de la langue française, défigure celle-ci. Il faut noter qu'une telle traduction a des antécédents. En traduisant l'Odyssée en 1781 puis l' Iliade en 1793, Voss cherche à modeler et élargir la langue allemande grâce à la métrique grecque (Berman 1984: 82, 224-225). De manière plus radicale, Hölderlin ([1800] 1985 et [1804] 1988) malmène le vers allemand grâce à la syntaxe grecque lorsqu'il traduit Sophocle et Pindare, et creuse dans l'épaisseur historique du lexique allemand pour en exhumer l'étymologie et en restituer l'étrangeté (Zuberbülher 1969).

\section{D'une défiguration l'autre : intraduire Oskar Pastior}

12 J'aimerais apporter ici une distinction importante. Pour ce qui concerne la traduction poétique, on peut distinguer trois formes de défiguration. Dans un premier cas, le texte à traduire met en jeu une écriture défigurée qui, pour cette raison, relève de l'intraduisible. Comment traduire, en effet, une écriture qui s'écarte des normes usuelles de la langue commune et qui, de fait, n'est plus transposable dans aucun autre idiome que son idiome propre et absolument singulier? Cette écriture induit alors, lorsque l'on tente de la traduire, une écriture seconde, dérivée et elle aussi 
intraduisible. C'est le cas des textes de Pierre Guyotat que j'ai évoqués plus haut, ou des poèmes de Cummings traduits par Jacques Demarcq (Cummings [1958] 1983).

Dans le deuxième type de défiguration, le texte d'origine respecte les usages linguistiques courants mais c'est le texte-cible, parce qu'il adopte un mode singulier de traduction, qui opère un acte de défiguration. C'est ainsi que Pierre Klossowski traduit Virgile ou que Friedrich Hölderlin traduit Sophocle et Pindare ${ }^{6}$. Frédéric Forte et Bénédicte Vilgrain se trouvent dans la même situation lorsqu'ils traduisent les Anagramgedichte d'Oskar Pastior, des poèmes où tous les vers sont des anagrammes du premier. En vis-à-vis du texte allemand, les traducteurs procurent deux versions de chaque poème : une première, littérale conforme à la grille anagrammatique du poème original (chaque vers traduit est l'anagramme du premier vers traduit, mais tente de restituer quelque chose du sens des vers d'origine) et une seconde, sémantique, où le sens du poème allemand est restitué sans effort pour anagrammatiser le texte. Enfin, des notes expliquent les choix de traduction des deux versions mais sur un mode cryptique qui constitue un quatrième poème (Pastior [1985] $2008: 12$ ).

Il existe enfin un troisième type de défiguration où le texte est d'emblée plurilingue : de fait, il met en échec les stratégies ordinaires de traduction et réclame plutôt une intraduction. C'est le cas des textes d'Oskar Pastior et de James Joyce que j'évoquerai plus loin. Cette troisième forme n'est pas radicalement différente de la première dans la mesure où ses effets à la lecture sont similaires. Chez Guyotat, par exemple, de nombreux patois et dialectes s'invitent sur la page, au cœur de la langue française ; on pourrait ainsi considérer que l'écriture de l'auteur relève elle aussi d'un plurilinguisme. Dans ces trois cas néanmoins, l'écriture (ou la traduction chez Hölderlin et Klossowski) est bel et bien défigurante, et produit à la fois de l'intraduisible et de l'illisible, soit parce qu'elle ne peut faire autrement, soit parce qu'elle porte, en puissance, un rapport singulier au langage. En reprenant la terminologie de Derrida dans «Des tours de Babel », je dirais que le texte défiguré est "traductible (übersetzbar) et intraduisible»: " comme aucun sens ne s'en laisse détacher, transférer, transporter, traduire dans une autre langue comme tel (comme sens), il commande aussitôt la traduction qu'il semble refuser » (1987a : 235). Derrida entend par là que les textes les plus intraduisibles sont aussi ceux qui, avec le plus d'insistance, appellent de leurs vœux la traduction ; celle-ci est leur «nécessité comme impossibilité » (208). Leur intraduisibilité met en évidence un rapport singulier au langage que la traduction fait apparaître avec éclat. Les essais de traduction en français du Krimgotische Fächer d'Oskar Pastior soulignent cette tension entre traductibilité et intraduisibilité. Ainsi de la «Ballade vom defektem Kabel » :

«Ballade du câble défectueux »

L'adafactas

Kveaunbl

Illtz crahoutz cataractaque-lment

Kascavldesk

ossabranx Brambl

ossa 'ndoth

Abwattez le

abwattez le

filiozon

Lo caskavlad

Lhabemus là?

Lhabemus 


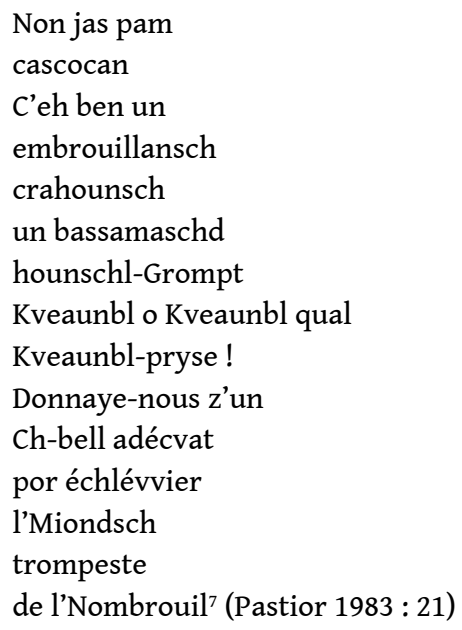

La traduction de Renate Kühn pour le numéro 16 de TXT prend appui sur les mots qui, pour un locuteur allemand, restent lisibles au milieu du chaos de syllabes des poèmes de Pastior. Ainsi, au deuxième vers, le «cow» de «Cowlbl» donne le «veau » de « Kveaunbl»; au troisième vers, Kühn traduit « rumplnz » par « crahoutz » tandis que «kataraktasch-lych» devient «cataractaque-lment». «Uotrfawls » devient « Kascavldesk » et «s-trumpltsch » devient « trompeste ». Le «s- » utilisé ici comme un préfixe s'invite, dans la traduction, en position appuyante après le « $\mathrm{e}$ ", comme une résurgence $\mathrm{du}$ « $\mathrm{s}$ » qui, après son amuïssement $\mathrm{au} \mathrm{XI} \mathrm{I}^{\mathrm{e}}$ siècle et son effacement au XVIII siècle, s'est changé en accent circonflexe. Sur le plan morphologique, le statut de «trompeste » est ambigu : il s'agit d'un substantif muni du suffixe « ste » autant qu'un verbe à la troisième personne du singulier, de même que «s-trumpltsch » hésite entre le verbe et le substantif avec affixes. Enfin le « Naawbl» du dernier vers fait entendre à la fois « Nabel» (« nombril») et « Nebel» («brouillard») au locuteur allemand. Cette hésitation vocalique, Kühn la restitue dans le mot-valise « Nombrouil ».

Les poèmes de Pastior convoquent ainsi des éléments figuratifs minimaux, tout droit issus de la pastorale et des églogues virgiliennes - le veau, la chute d'eau, la nudité édénique des corps - mais le contour de ces objets est comme biffé et maculé de l'intérieur par le travail non figuratif du texte et la poussée d'autres idiomes ou de racines appartenant à plusieurs langues à la fois, depuis le cœur même des mots. La traduction ne rend pas ici le texte plus illisible qu'il ne l'est déjà. Simplement, le poème de Pastior fait d'elle moins un processus de transposition d'une langue à l'autre qu'un mécanisme où s'inventent, par d'autres moyens, d'autres formes d'illisibilités.

Aussi les traductions possibles d'un même poème de Pastior diffèrent-elles radicalement les unes des autres. En 2014, j'ai publié dans la revue Vacarme une traduction alternative de la « Ballade vom defektem Kabel », fondée sur de tout autres principes (Pastior 2014 : 29). Là où la traduction de Renate Kühn visait à assurer une ressemblance minimale au travers de certains mots tout en maintenant des points d'opacité, de manière à donner des prises au lecteur et intensifier le contraste du lisible et de l'illisible, j'ai plutôt cherché à déplier chacune des dissemblances du texte d'origine pour restituer au lecteur français un équivalent de l'expérience que fait le lecteur allemand devant ces poèmes.

«Ballade du kowlbble défectueux "

Ajouzyfés

Clâochebale

Ed wascarmeonnair cataractsacqueuhx 


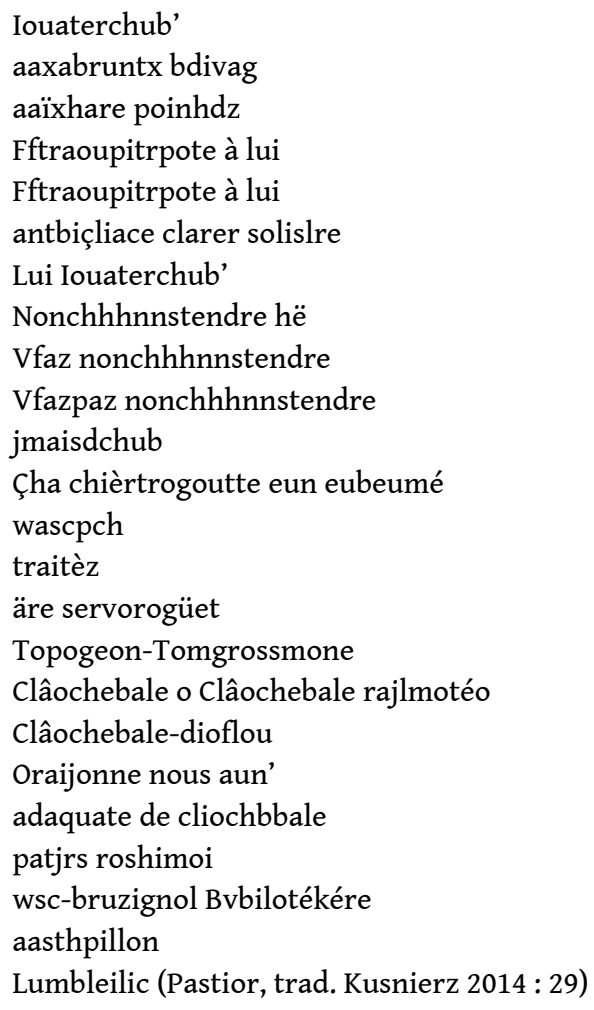

Alors que Renate Kühn laissait certains mots intacts, j'ai proposé une traduction possible pour chacune des racines identifiables. Surtout, j'ai tâché de réinjecter de la dissemblance et de la défiguration dans des proportions équivalentes à celles du texte d'origine. Voici ce que j'écrivais alors pour expliquer ma démarche :

À partir d'une enquête étymologique dans la pluralité et l'épaisseur des idiomes, j'ai voulu restituer l'expérience de la traversée et de la confusion linguistiques qui s'exposent sur la page et que le texte fait subir au lecteur. Je me suis donc muni de dictionnaires bilingues pour traduire en français, tout en gardant quelque chose de leur langue d'origine, les mots et les racines qui apparaissent dans le texte. Il fallait restituer à la fois son caractère incompréhensible et sa manière de faire entendre malgré tout des lambeaux de signification, sans oublier les interférences des différents idiomes entre eux, puisque certaines racines ressortissent à plusieurs langues. À ma grande surprise, cette méthode s'est avérée fonctionnelle et a permis de révéler, par bribes, des réseaux de sens instables. C'est donc que l'enquête étymologique permettait bel et bien d'assurer la traduction, comme on le dit des alpinistes, sans toutefois procurer de certitude solide. [...] Il s'est ainsi agi, en traduisant, de faire entendre au lecteur français la même production de significations confuse, fragmentaire et défigurée que le lecteur allemand entend dans le texte d'origine. (Pastior 2014 : 32-33)

Renate Kühn visait une certaine clarté de lecture grâce à une traduction ressemblante ; la traduction de Vacarme vise l'équivalence des expériences au travers de la dissemblance.

Au regard des conceptions traditionnelles de la traduction, une traduction défigurante est toujours ratée ${ }^{8}$. Soit parce qu'au lieu de transposer un texte de manière lisible dans la langue cible, elle en manque le sens et produit une écriture illisible, soit parce que, dans l'écriture défigurée, il ne reste plus rien à traduire, comme si la défiguration épuisait la traduisibilité du texte tout en amplifiant sa traductibilité ${ }^{\text {. Dans le premier }}$ cas, l'écrivain marque l'absolue singularité de son espace linguistique en s'assurant de son intraduisibilité. Dans le deuxième cas, le traducteur « dégonde la langue », comme 
le dit Beckett : que l'on pense à Hölderlin lorsqu'il traduit Sophocle et Pindare ou à Klossowski lorsqu'il traduit Virgile, ou encore à Frédéric Forte et Bénédicte Vilgrain lorsqu'ils traduisent les Anagrammgedichte d'Oskar Pastior. Dans le troisième cas, le texte, parce qu'il mobilise plusieurs idiomes, est déjà une écriture traductrice. La dimension plurilingue du Krimgotische Fächer de Pastior ou du Finnegans Wake de Joyce neutralise l'opération traductrice, au moins dans son acception traditionnelle. Je reviendrai plus loin sur cette question.

\section{Matérialisme de la traduction}

Revenons pour le moment à l'adage italien, «traduttore, traditore ». Plutôt que d'énoncer la malédiction de la traduction, il me semble qu'il ouvre pour celle-ci un champ de possibilités. Au lieu d'y lire une condamnation, je préfère considérer que l'adage définit l'échec et la trahison comme la condition de possibilité de la traduction et même comme sa chance. L'infidélité ne serait pas alors un défaut inhérent à la traduction mais bien l'origine d'une invention nouvelle, la possibilité d'une bifurcation au cœur de la langue, comme le montre Daniel Heller-Roazen (2014 : 9-17). Il faut alors considérer la trahison moins comme une incomplétude que comme la production d'un nouveau texte décollé du premier. C'est ce que montrent à nouveau, par excellence, les traductions des poèmes d'Oskar Pastior ou de l'Énéide par Klossowski : la traduction y est un travail de création poétique au sens fort. Qui plus est, dès lors que toute traduction trahit, ne peut-on pas plutôt penser qu'une traduction défigurante excède de très loin les traditionnelles questions de fidélité, de réussite ou d'échec de l'opération traductrice ? Il faut ainsi déplacer notre regard sur la traduction et écouter Henri Meschonnic pour qui un «traducteur [...] donne à entendre ce que fait un poème et pas seulement ce qu'il dit » (Meschonnic 2006 : 9). Autrement dit, la traduction agit concrètement sur le texte d'origine, dans un geste matérialiste ; elle le réinvente et lui offre une existence alternative - quand la traduction traditionnelle prolonge, dans d'autres langues, l'existence du texte premier.

19 La défiguration, en somme, serait alors l'opération qui arrache la traduction au gardefou de la fidélité et à son cortège de mesures coercitives. Dans la défiguration, la traduction s'occuperait ainsi moins de convertir du sens d'une langue à une autre que de le produire librement, exempté de l'obligation d'interpréter le texte d'origine et de restituer avec exactitude sa signification. Dans un hommage à son traducteur Nestor Ibarra - qui préférait traduire plutôt qu'écrire -, Borges écrit que ce dernier considère la traduction comme une "étape plus avancée » de l'écriture (Charbonnier 1967: 14 ; Borges 2010 : 1505). Il faut comprendre ici que la traduction est une écriture qui ne s'occupe plus que de la langue, à l'exception de tout le reste. La défiguration réaliserait ainsi ce rêve borgésien d'une traduction enfin libérée des contraintes du sens pour ne plus se consacrer qu'à la production de la langue, ou plutôt à sa productivité, pour emprunter à Barthes un concept fécond. Mais à quoi bon cette productivité de la langue?

20 Je formulerai de la façon suivante une esquisse de réponse. Puisque la traduction défigurante passe outre l'obligation de respecter ou d'interpréter la signification du texte d'origine, elle est une anti-herméneutique. Les conséquences politiques en sont importantes. Les auteurs réunis autour de TXT partagent tous la conviction que le travail expérimental de la langue revêt une fonction politique. Il me semble que c'est 
dans la défiguration et l'intraduction - soit la confrontation traduisante avec l'intraduisible - que cette fonction politique apparaît le plus nettement. D'après Dominique Fourcade, l'écriture poétique expérimentale opère "dans un opaque profond", c'est-à-dire qu'elle arrache le langage aux idéologies et aux langages stéréotypés que nous recevons en partage de la société (Gleize 2011:28). Si, comme l'écrit Ponge, la poésie est «l'art de résister aux paroles, l'art de ne dire que ce que l'on veut dire, l'art de les violenter et de les soumettre » (1999: 193), alors elle est bien un geste émancipateur. Et l'indéchiffrable, l'illisible et l'intraduisible sont le moyen de cette émancipation. Jean-Pierre Verheggen parle ainsi du «langagement » (1994: 18) comme d'une action menée sur l'ordre sociopolitique par et à travers le langage. Et Jean-Marie Gleize, commentant le concept de Verheggen, écrit qu'il désigne une «subversion de l'ordre des représentations par la langue, par le travail sur la langue ou les langues ", avant d'affirmer que "toute écriture véritable, en tant que mécriture ou torsion poétique de la langue, [est] par définition illisible ou monstrueuse » (Gleize 2011 : 39). On voit ici comment les concepts de traduction et de défiguration trouvent un terrain d'entente dans les pensées de Verheggen et de Gleize ${ }^{10}$.

\section{L'intraduction, ou la dissémination babélienne des idiomes}

Ce travail sur les langues, il revient évidemment à la traduction de l'accomplir en faisant entendre leurs différences ; soit en passant simplement de l'une à l'autre, soit en les juxtaposant dans une dissémination babélienne opérée à même la page, telle que la décrit Derrida dans La Dissémination:

Tour de Babel où les langues et écritures multiples se heurtent et passent les unes dans les autres, se transforment et s'engendrent depuis leur altérité la plus irréconciliable, la plus affirmée aussi car la pluralité ici n'a pas de fond et n'est pas vécue comme négativité, dans la nostalgie de l'unité perdue. (Derrida 1972 : 379)

Cette tour de Babel dont parle Derrida, ce pourrait être la colonne du texte d'Oscar Pastior, agrégat de langues européennes en lambeaux que le travail rythmique détoure et découpe dans l'espace pluriel des idiomes, de manière à le rendre à la fois intraduisible et illisible. Ce pourrait être aussi la langue de synthèse qu'Ernst Jandl élabore dans Falamaleikum et Stanzen, à la fois primitive et en ruines, arrivée au bout de son histoire et désormais impossible à transposer dans un autre idiome.

minz den gaawn

bill den baud

minz den gaawn

bill den baud

kittl koo

kittl koo

minz den gaawn

gnaz den eschn

ruttl znop (Jandl $1983: 80$ )

maunchmoe howe des gfüü

de wööd schded fuan untagaung

daun dafaunge me und sog ma

des gütt nua fia mii (Jandl 1992 : 78)

De fait, écrire ou traduire de tels textes consiste plutôt à intraduire : à la fois parce que l'écriture est d'emblée plurilingue et parce qu'il n'est plus possible de faire passer une langue unique dans une autre langue unique en gardant intacts, le plus possible, le sens 
et la forme du texte, c'est-à-dire en conservant l'identité du texte d'origine. De fait, l'intraduction, plus qu'une transposition d'une langue à l'autre, se donne comme une dissémination du texte dans l'infinité des langues.

Dans « Des tours de Babel », Derrida (1987a : 207-208) se demande comment penser « la possibilité pour des langues d'être impliquées à plus de deux dans un texte. Comment traduire un texte écrit en plusieurs langues à la fois ? [...] Et si l'on traduit par plusieurs langues à la fois, appellera-t-on cela traduire? ». Le philosophe montre alors que Joyce, en écrivant Finnegans Wake en plusieurs langues, déjoue la question de l'unité linguistique de la littérature et interdit de "déterminer rigoureusement l'unité et l'identité d'une langue, la forme décidable de ses limites" (207-209). C'est alors une guerre qui fait rage dans le langage, écrit-il, une guerre résumée par ce fragment de texte, qui rassemble "un nombre incalculable de fils phoniques et sémantiques " (207) : " And shall not Babel be with Lebab? And he war. And he shall open his mouth and answer: I hear, O Ismael, how they laud is only as my loud is one " (Joyce [1939] 2000 : 258). Mais cette guerre met fin à « l'impérialisme linguistique» (Derrida 1987a : 210) car elle substitue la nécessité de la traduction à celle de l'unité comme loi de la littérature. En faisant littéralement éclater l'unité entre l'œuvre et la langue mais aussi entre la langue et le sol, Finnegans Wake opère un redécoupage à la fois symbolique et virtuel de la communauté des lecteurs (Derrida 1987b : 16-17, 35-41). Celle-ci n'est plus circonscrite, de pays en pays, par l'usage et la maitrise d'une langue maternelle; elle traverse les frontières, abolit l'incompréhension mutuelle des langues et transcende les groupes de locuteurs. Le texte joycien n'abolit pas la confusion post-babélienne ni ne dépasse dialectiquement la pluralité des idiomes; il est au contraire un mouvement sans relève ${ }^{11}$ ni arrêt, qui refuse à la fois de se réduire à la somme des langues qu'il contient et de constituer une seule langue synthétique et syncrétique. Finnegans Wake est un poumon qui enfle et se contracte sans cesse entre la ligne d'horizon d'une langue unique et la constellation infinie des idiomes. Il est ce " point sans doute infiniment petit - à la limite de la traduction » dont parle encore Derrida : «l'intraduisible pur et le traductible pur y passent l'un dans l'autre - et c'est la vérité, "elle-même matériellement" » (1987a : 223) parce que la langue y est constamment en traduction en même temps qu'elle la repousse. Le philosophe le dit encore autrement: en ce point infiniment petit, la langue - qui se confond ici avec la vérité - se trouve "au-delà de toute Übertragung [transcription] et de toute Übersetzung [traduction] possibles » (228). Cette vérité n'est plus l'adéquation entre l'original et sa traduction; elle est désormais " le langage pur en lequel le sens et la lettre ne se dissocient plus » (228), c'est-à-dire un langage dans lequel la traduction, devenue impossible, n'est plus capable de transposer $\mathrm{du}$ sens dans un autre langage.

\section{Politique de l'intraduction}

C'est ici que se joue la fonction politique de l'intraduction, de manière à la fois positive et négative. Le texte défiguré est comme un rébus à déchiffrer, une partition à faire sonner aux risques et périls de l'interprète et de l'auditeur. C'est ainsi que JeanFrançois Mura dit de sa traduction de Jandl qu'elle est une transcription: transcrire consiste à « écrire de la musique pour un instrument autre que celui en vue duquel elle a été écrite " (Mura 2013 : 28). Or cette transposition court le danger de rompre l'unité entre la partition et l'instrument, de même que l'entente entre le lecteur et l'auteur. 
Mais ce risque ouvre aussi la possibilité d'une communauté, et avec elle une politique. En refondant la langue, l'intraduction reconfigure l'espace de sens qu'elle dessine pour nous; elle élabore un nouveau "partage du sensible» (Rancière $2000: 24-25)$ qui prélude à un rapport renouvelé au commun. Le partage du sensible désigne l'appropriation collective des manières de percevoir et de penser. Parce qu'elles confrontent le lecteur à une pluralité d'autres langues, parlées ou non, la poésie de Pastior et celle de Jandl lui proposent une autre manière de percevoir le monde. À d'autres manières de sentir, d'autres manières de penser et de vivre: de proche en proche, la poésie qui redéfinit la perception du lecteur en le faisant changer d'espace linguistique se propose aussi de transformer ses conditions d'existence et lui permet de développer de nouvelles formes de conscience.

Sur le mode négatif, la traduction défigurante révèle la dissemblance entre les langues bien plus qu'elle ne révèle leur parenté, comme le voulait Benjamin dans « La tâche du traducteur " ([1923] 2000: 249). Et si cette tâche consiste bien à «faire mûrir la semence d'un pur langage ${ }^{12}$ ", le penseur ajoute aussitôt que "de cette tâche [...], il semble impossible de jamais s'acquitter ${ }^{13}{ }$ (255). C'est donc bien que ce pur langage est en réalité le revers inaccessible et fantomatique d'une dissemblance des idiomes qui, elle, tient à la matière même du langage. Le fossé qui sépare les langues les unes des autres nous donne l'idée du langage pur mais la traduction - qui plus est lorsqu'elle défigure - nous renvoie sans cesse à leur irréductible dissemblance. Au début de L'Absolu comparé, Éric Dayre écrit :

La traduction est ce qui arrive à la langue. [...] Une traduction, c'est-à-dire l'insistance indéfinie d'une autre écriture face à la langue maternelle, est déstabilisante pour une théorie poétique qui se voudrait fondée sur l'identité de la langue, et sur l'idée selon laquelle le style serait d'abord l'affaire d'une seule langue à la fois. $(2009: 5-7,18-20)$

Dayre signifie par là qu'en faisant exister la dissemblance entre les langues dans l'espace littéraire, la traduction est à penser comme une activité qui délie, en basse continue, la langue et le sol. En exploitant cette puissance que la traduction contient en réserve, l'intraduction - entendue comme traduction défigurante et confrontation traduisante avec l'intraduisible - déjoue ainsi l'«homo-hégémonie» de la langue maternelle unitaire (Derrida $1996: 45,55-69)$ et défait les assignations à une origine ou à un sol. En somme, l'intraduction joue le même rôle, dans l'ordre des textes, que Dieu dans la Bible lorsqu'il détruit la tour de Babel et, avec elle, la langue universelle : « il disperse la filiation généalogique [et] rompt la lignée" (Derrida 1987a: 207). L'intraduction rompt l'unité d'une langue et d'un sol, donc le concept même de langue nationale, pour faire valoir une idée de la langue qui ne se confonde pas avec celle d'ethnie mais qui propose de réinventer la communauté qu'elle recouvre. Elle est donc bien un acte d'émancipation politique car cette négation ouvre dialectiquement au locuteur la possibilité d'une réinvention symbolique de son identité. Pour lire Pastior et Jandl, je dois apprendre à lire et à parler dans une autre langue que ma langue maternelle, une langue qui n'appartient à aucun territoire et aucune nation mais qui accueille tous ses locuteurs potentiels. Je quitte mon identité mais n'en adopte aucune autre que définirait un sol ou un État et dans ce vide, cette indéfinition réalisée par et dans la langue, c'est le livre qui invente pour moi une identité mouvante, instable et qui miroite dans la réunion de tous ses lecteurs. Ainsi les poètes qui défigurent accomplissent-ils aussi, généreusement, le don des langues. 


\section{BIBLIOGRAPHIE}

Artaud, Antonin, 1987, Euvres complètes, volume 4, Paris, Gallimard, coll. « Blanche ».

Barthes, Roland, 1984, Le Bruissement de la langue. Essais critiques IV, Paris, Seuil.

Barthes, Roland, [1973] 2002, « Texte (théorie du) », dans EEuvres complètes. Livres, textes, entretiens, volume 4, Paris, Seuil, p. 443-459.

Bataille, Georges, 1955, « Préface », dans D. A. F. de Sade, Justine ou Les Malheurs de la vertu [1791], Paris, Jean-Jacques Pauvert, p. VII-XXXVII.

Benjamin, Walter, [1923] 2000, « La tâche du traducteur », dans Euvres, vol. 1, trad. Maurice de Gandillac, Rainer Rochlitz et Pierre Rusch, Paris, Gallimard, coll. « Folio Essais », p. 244-262.

Berman, Antoine, 1984, L'Épreuve de l'étranger. Culture et traduction dans l'Allemagne romantique, Paris, Gallimard, coll. « Les Essais ».

Bhabha, Homi K., 1994, The Location of Culture, New York, Routledge.

Borges, Jorge Luis, 2010, Euvres complètes, volume 1, édition établie, présentée et annotée par Jean-Pierre Bernès, Paris, Gallimard, coll. « Bibliothèque de la Pléiade ».

Burroughs, William S., 1959, The Naked Lunch, Paris, Olympia Press, coll. « The Traveller's Companion Series ».

Burroughs, William S., 1961, The Soft Machine, Paris, Olympia Press, coll. « The Traveller's Companion Series ».

Burroughs, William S., 1962, The Ticket That Exploded, Paris, Olympia Press, coll. « The Traveller's Companion Series ».

Burroughs, William S., 1964, Nova Express, New York, Grove Press.

Burroughs, William S., [1970] 1974, Révolution électronique, suivi de Time et de Étoile morte, trad. Jean Chopin, Paris, Champs Libre.

Burroughs, William S., 1979, Le Job. Entretiens avec Daniel Odier, Paris, Belfond, coll. « Entretiens ». Casanova, Pascale, 1999, La République mondiale des Lettres, Paris, Seuil.

Casanova, Pascale, 2015, La Langue mondiale. Traduction et domination, Paris, Seuil, coll. « Liber ».

Casanova, Pascale et Samoyault, Tiphaine, 2005, « Entretien sur La République mondiale des Lettres ", dans C. Pradeau et T. Samoyault (dir.), Où est la littérature mondiale ?, Saint-Denis, Presses universitaires de Vincennes, coll. « Essais et savoirs », p. 139-150.

Charbonnier, Georges, 1967, Entretiens avec Jorge Luis Borges, Paris, Gallimard.

Chiurazzi, Gaetano, 2013, « Entre totalisation et incomplétude : le pouvoir émancipateur de la traduction », Noesis, $n^{\circ}$ 21, La philosophie, la traduction, l'intraduisible, p. 89-105.

Cummings, Edward Estlin, [1958] 1983, 95 poèmes, trad. Jacques Demarcq, Paris, Flammarion, coll. « Textes».

Dayre, Éric, 2009, L'Absolu comparé. Littérature et traduction. Une séquence moderne : Coleridge, De Quincey, Baudelaire, Rimbaud, Paris, Hermann.

Deleuze, Gilles, 1993, Critique et clinique, Paris, Minuit, coll. « Paradoxe ». 
Demarcq, Jacques, 2008, Les Zozios, Caen, Nous.

Demarcq, Jacques, 2017, Suite Apollinaire, Barjols, Plaine page, coll. « Calepins ».

Derrida, Jacques, 1967, L'Écriture et la Différence, Paris, Seuil, coll. « Tel quel ».

Derrida, Jacques, 1972, La Dissémination, Paris, Seuil, coll. « Tel quel ».

Derrida, Jacques, 1974, Glas, Paris, Galilée, coll. « Digraphe ».

Derrida, Jacques, 1987a, « Des tours de Babel », dans Psyché. Inventions de l'autre, Paris, Galilée, coll. « La philosophie en effet », p. 203-235.

Derrida, Jacques, 1987b, Ulysse gramophone. Deux mots pour Joyce, Paris, Galilée, coll. « La philosophie en effet ».

Derrida, Jacques, 1996, Le Monolinguisme de l'autre, ou la prothèse d'origine, Paris, Galilée, coll. «Incises ».

Faye, Jean-Pierre, 1968, « Montage, production », Change, $\mathrm{n}^{\circ}$ 1, Le montage, p. 5-13.

Gleize, Jean-Marie, 2011, « Opacité critique », dans J.-C. Bailly, J.-M. Gleize et al., « Toi aussi, tu as des armes ». Poésie \& politique, Paris, La Fabrique, p. 27-44.

Grossman, Évelyne, 2004, La Défiguration. Artaud, Beckett, Michaux, Paris, Minuit, coll. « Paradoxe ». Guyotat, Pierre, 2000, Progénitures, Paris, Gallimard.

Hegel, Georg Wilhelm Friedrich, [1817] 1970, La Science de la logique, trad. Bernard Bourgeois, Paris, Vrin, coll. «Bibliothèque des textes philosophiques ».

Heller-Roazen, Daniel, 2014, Dark Tongues: The Art of Rogues and Riddlers, New York, Zone Books.

Hölderlin, Friedrich, [1800] 1985, Sämtliche Werke. Historisch-kritische Ausgabe in 20 Bänden und 3 Supplementen, volume 15, Pindar, Francfort-sur-le-Main, Stroemfeld, Roter Stern.

Hölderlin, Friedrich, [1804] 1988, Sämtliche Werke. Historisch-kritische Ausgabe in 20 Bänden und 3 Supplementen, volume 16, Sophokles, Francfort-sur-le-Main, Stroemfeld / Roter Stern.

Jandl, Ernst, 1983, Falamaleikum. Gedichte und Bilder, Darmstadt, Luchterhand.

Jandl, Ernst, 1992, Stanzen, Hamburg, Luchterhand.

Joyce, James, [1939] 2000, Finnegans Wake, Harmondsworth, Penguin Books.

Klossowski, Pierre, [1964] 2015, « Préface », dans Virgile, Énéide, trad. Pierre Klossowski, Lyon, Trente-trois morceaux, p. 15-16.

Kristeva, Julia, 1969, Sēmeiōtiké. Recherches pour une sémanalyse, Paris, Seuil, coll. « Tel quel ».

Kusnierz, Mathias, 2012, « Hölderlin traducteur : mythos, mutisme », Textuel, nº 68, Mythe(s): Construction, traduction, interprétation, p. 71-81.

Ladmiral, Jean-René, 1979, Traduire. Théorèmes pour la traduction, Paris, Payot, coll. « Petite bibliothèque Payot $"$.

Ladmiral, Jean-René, 2014, Sourcier ou cibliste, Paris, Les Belles Lettres, coll. « Traductologiques ». Meschonnic, Henri, 2006, Vivre poème, Liancourt, Reims, Dumerchez, coll. « Double Hache ».

Mura, Jean-François, 2013, « Traduction, transcription ou interprétation ? », dans E. Jandl, Luminesens, trad. Jean-François Mura, Mont-de-Laval, L'Atelier du Grand Tétras, coll. « Entre deux rives », p. 27-30. 
Ortega y Gasset, José, [1940] 1964, Miseria y esplendor de la traducción, dans Obras completas, Madrid, Revista de Occidente, volume 5.

Pastior, Oskar, 1978, Der krimgotische Fächer. Lieder und Balladen. Mit 15 Bildtafeln des Autors, Erlangen, Verlag Klaus G. Renner.

Pastior, Oskar, 1983, « Ballade du câble défectueux », trad. Renate Kuhn, TXT, n $16:$ La fiction délie les langues, p. 21.

Pastior, Oskar, [1985] 2008, 21 Poèmes-anagrammes d'après Hebel, trad. Frédéric Forte et Bénédicte Vilgrain, Courbevoie, Théâtre typographique.

Pastior, Oskar, 2014, « wsc-bruzignol Bvbilotékére », trad. Mathias Kusnierz, Vacarme, $\mathrm{n}^{\circ}$ 69, p. 24-33.

Ponge, Francis, 1999, Cuures complètes, vol. 1, édition établie sous la direction de Bernard Beugnot, Paris, Gallimard, coll. «Bibliothèque de la Pléiade ».

Priessnitz, Reinhard, [1978] 2015, 44 poèmes. Poésie complète, trad. Alain Jadot, Caen, Nous, coll. « Grmx ».

Prigent, Christian, 1989, La Langue et ses monstres, Montpellier, Cadex, coll. « L'Ostiaque ».

Prigent, Christian, 1992, Être moderne, est-ce être illisible ?, Reims, Noria, coll. « Cahiers de Noria ».

Prigent, Christian, 1994, À quoi bon encore des poètes ?, Valence, ERBA, coll. « 222 ».

Prigent, Christian, 2004, Ne me faites pas dire ce que je n'écris pas. Entretiens avec Hervé Castanet, Saussines, Cadex, coll. « David ».

Prigent, Christian, 2009, Christian Prigent, quatre temps. Rencontre avec Bénédicte Gorillot, Paris, Argol, coll. « Les Singuliers ».

Prigent, Christian, 2014, « Du sens de l'absence de sens », dans B. Gorillot et A. Lescart (dir.), L'Illisibilité en questions, Villeneuve-d'Ascq, Presses Universitaires du Septentrion, coll. « Littératures », p. 31-36.

Prigent, Christian, 2015, « Opéra Priessnitz », dans R. Priessnitz, 44 poèmes. Poésie complète, trad. Alain Jadot, Caen, Nous, coll. « Grmx », p. 7-21.

Rancière, Jacques, 2000, Le Partage du sensible. Esthétique et politique, Paris, La Fabrique.

Rimbaud, Arthur, 2009, « À Paul Démeny, 15 mai 1871 », dans Cuvres complètes, édition établie par André Guyaux avec la collaboration d'Aurélia Cervoni, Paris, Gallimard, coll. « Bibliothèque de la Pléiade ».

Verheggen, Jean-Pierre, 1994, Ridiculum vitae, Paris, La Différence, coll. « Littérature ».

Virgile, 2015a, Énéide, trad. Olivier Sers, Paris, Les Belles Lettres.

Virgile, [1964] 1989, L'Énéide, trad. Pierre Klossowski, Marseille, André Dimanche, coll. « Ryôanji ».

Virgile, [1964] 2015b, Énéide, trad. Pierre Klossowski, Paris, Trente-trois morceaux.

Zuberbülher, Rolf, 1969, Hölderlins Erneuerung der Sprache aus ihren etymologischen Ursprüngen, Berlin, Erich Schmidt. 


\section{NOTES}

1. Fondée en 1969 et disparue en 1993 avant sa résurrection en 2018, TXT se veut une alternative provinciale à $\mathrm{Tel}$ Quel, alors tête de pont des avant-gardes littéraires (Prigent 2004: 97-99). TXT conçoit le travail sur le langage dans une perspective carnavalesque (Prigent 2004: 98 et 105). Dans la pratique poétique, celui-ci devient l'espace au sein duquel renverser toutes les valeurs traditionnelles de la littérature : la beauté, la production du sens, l'inscription du texte dans l'histoire de la littérature et dans la culture savante, l'œuvre comme objet sacré et respectable, fondant l'autorité de son producteur et imposant sa loi au lecteur (Barthes [1973] 2002).

2. L'adjectif "théologique» renvoie ici à ce que Barthes a nommé ailleurs une «métaphysique [...] de la vérité » ([1973] $2002:$ 444), à savoir une conception de la littérature qui place en son cœur l'auteur et l'expression de sa vision du monde. Cette métaphysique sous-tend la pratique idéaliste bourgeoise de l'écriture, fondée sur la psychologie et le signifié plutôt que sur le travail du signifiant. C'est contre elle que travaillent les avant-gardes poétiques.

3. Cette formule de Rimbaud vient de la lettre à Paul Démeny du 15 mai 1871, dite « lettre du Voyant » $(\mathrm{NdA})$.

4. "Excisum Euboica latus ingens rupis in antrum, / quo lati ducunt aditus centum, ostia centum; / unde ruunt totidem uoces, responsa Sibylloe. / Ventum erat ad limen, cum uirgo. "Poscere fata / tempus" ait; "deus, ecce, deus!" Cui talia fanti / ante fores subito non uoltus, non color unus, / non comptae mansere comae; sed pectus anhelum, / et rabie fera corda tument ; maiorque uideri, / nec mortale sonans, adflata est numine quando / iam propiore dei " (Virgile 2015a : 266 ; le traducteur a ajouté la ponctuation pour une lecture plus commode).

5. On peut se reporter à l'édition de 1989 publiée chez André Dimanche, qui comporte un glossaire d'une quarantaine de mots (Virgile 1989).

6. Sur cette question, je me permets de renvoyer le lecteur à mon article «Hölderlin traducteur : mythos, mutisme » (Kusnierz 2012).

7. «Adafactas / Cowlbl / Ed rumplnz kataraktasch-lych / Uotrfawls / aachabrawnkts Brambl / aachr dohts ... // Schlochtehz ihm / schlochtehz ihm / ehs klaren Zohn // Ihn Uotrfawls // Humrem hä? / Do humrem / Nodo humrem / Kaineschfawls // Ehs ischtolt ain däfäktäs / rumpltsch / traktaz / ä nedderschtilchz / Rompl-Grompt // Cowlbl o Cowlbl wottä / Cowlbl-gotz! // Gehbät uns ain / adakuats Ch-bell / ntmr hiechffn / s-trumpltsch Bvchuelltr / aasm / Naawbl » (Pastior 1978 : 13).

8. Notons qu'il y aurait tout un travail d'archéologie à mener pour nuancer cette affirmation qui vaut surtout pour l'espace francophone moderne. La tradition de traduction germanophone, de Luther à Humboldt en passant par Goethe, obéit à des principes bien différents de formation (Bildung) et d'élargissement (Erweiterung) de la langue allemande (Berman 1984 : 61-71 et 226-249).

9. Je m'en remets de nouveau à Derrida pour préciser les notions de traduisibilité et de traductibilité. La première désigne la possibilité de transposer le sens du texte dans une autre langue tandis que la seconde indique l'appel du texte à être traduit, son à-traduire. Selon le philosophe, plus le texte résiste à la traduction, plus il demande à être traduit : « ce qui reste intraduisible est au fond la seule chose à traduire, la seule chose traductible. L'à-traduire du traductible ne peut être que l'intraduisible » (1987b : 59-60). 
10. Remarquons toutefois que Gleize (27-29) évoque, dans ce même texte, la possibilité d'un travail politique sur le langage fondé sur son exposition critique plutôt que sur sa subversion. L'illisibilité n'est donc pas la seule voie pour élaborer un rapport politique au langage, lequel ne s'oppose pas systématiquement aux normes de la langue.

11. Derrida, à la suite de Lacan, traduit par "relève » le mot allemand «Aufhebung ", concept de Hegel qui désigne le moment du dépassement dans le processus dialectique (Hegel [1817] 1970 : 530 ; Derrida 1967 : 375-381, 404 ; 1974).

12. "den Samen reiner Sprache zur Reife zu bringen ».

13. « diese Aufgabe [...] scheint niemals lösbar ».

\section{RÉSUMÉS}

Je propose dans cet article une approche historiquement située du problème de l'intraduisible poétique et je me concentre sur des écritures et des traductions "défigurantes ", à travers les textes d'Ernst Jandl, d'Oskar Pastior et d'Edward E. Cummings. Je montre que le concept d'intraduction cristallise une conception politique de la littérature, qui engage le risque de l'illisibilité. En refondant la langue, l'intraduction reconfigure l'espace de sens que celle-ci dessine pour nous ; elle élabore un nouveau partage du sensible, prélude à un rapport renouvelé au commun. La question de l'intraduisible est ainsi profondément liée à l'aventure des avantgardes poétiques.

In this paper I suggest an historical approach of the poetical untranslatable. I am focusing on disfigurating poetics and translations as offered in the works of Ernst Jandl, Oskar Pastior and Edward E. Cummings. I suggest that "untranslating" bears a political conception of writing and literature, based on the risk of being illegible. By inventing tongues anew, untranslating is remaking the space and the borders of meaning and signification that tongues are setting for us. Untranslating suggests a new distribution of the sensible which prelude to a renewed relationship with the commons. Untranslatable as a matter of poetics is thus deeply bound to the avant-garde's poetical adventure.

\section{INDEX}

Keywords : translation, disfiguration, avant-garde, untranslatable, translatability, illegibility, dissemination, politics, language

Mots-clés : avant-garde, traduction, défiguration, intraduisible, traductibilité, illisibilité, dissémination, politique, langage

\section{AUTEUR}

\section{MATHIAS KUSNIERZ}

Université de Paris, CERILAC 